Discussion This novel T-piece pull technique for the removal of $\mathrm{BB}$ is a simple endoscopic procedure, using equipment that is readily available in most endoscopy departments. The procedure time is much shorter than more complex endoscopic techniques, such as ESD. Lesions which have a $2 \mathrm{~b}$ Paris classification indicate a deeply buried bumper and should be considered for a different approach to removal.

\section{PWE-007 TYPE 2 INTESTINAL FAILURE- A LARGE TERTIARY HOSPITAL EXPERIENCE}

Thomas Hollingworth*, Lucy Bakewell, Elizabeth Buse, Emily Clarke, Zillah Leach, Priya Mistry, Carl Richardson, Charlotte Rutter, Andrew King, Trevor Smith. University Hospital Southampton, Southampton, UK

\subsection{6/gutjnl-2019-BSGAbstracts.338}

Introduction Type 2 intestinal failure (IF) is a prolonged ( $>28$ days), acute condition requiring complex multi-disciplinary (MDT) care and intravenous (IV) supplementation, either in hospital or through the provision of home parenteral nutrition (HPN). Without careful MDT management, these patients can progress to type $3 \mathrm{IF}$, requiring lifelong IV support with associated morbidity. NHS England estimates that there will be an increase in the provision of HPN at a rate of approximately $20 \%$ per annum, largely as a consequence of the increase in type 2 IF.

The aim of this study was to assess the efficacy of IF surgery that we provide in our tertiary referral centre in reducing the need for ongoing IV support. Secondary end points included the reason that IV support was required, the length of time from commencing IV support to surgery and the underlying pathology that led to the IF.

Methods A prospectively kept database of all patients receiving HPN who underwent IF surgery between September 2009 and July 2018. The notes of these patients were assessed with the dates or referral, length of IV support, reason for IV support, underlying pathology and IV support required following surgery recorded.

Results 77 operations were performed on 76 patients with a median age at the time of operation of 57 (range 1-1). 64 patients received HPN whilst 12 received IV fluids. These patients required IV support prior to an IF laparotomy due to fistulae (31), high output stoma (26), abdominal sepsis (15), and bowel obstruction (5). The underlying pathology that led to their presentation includes inflammatory bowel disease (26), vascular (13), surgical complications (10), malignancy (7), diverticular disease (7), Volvulus (4), Radiotherapy (3), adhesions (2), perforation (2), pancreatitis, TB and in one case unknown. The median time from starting IV support to the date of surgery was 218 days (range 10-67) Of these 92\% were able to stop their HPN and $75 \%$ had their home IV fluids stopped. 5 patients had their TPN reduced to intravenous fluids and 8 continued with their previous home intravenous support

Conclusions Surgery for type $2 \mathrm{IF}$, in selected patients who have close MDT management can lead to a significant reduction in the requirement for home IV support, reducing the associated morbidity and mortality. The MDT needs to closely support patients nutritionally, psychologically and physically, allowing time for recovery from the initial insult before embarking on further surgery.

\section{PWE-008 DOSING, DURABILITY OF HAEMOGLOBIN RESPONSE AND SAFETY OF IRON ISOMALTOSIDE IN PATIENTS WITH GASTROINTESTINAL DISEASES}

Amanda Thomas, Vida Cairnes, Sean Mole, Claire Elworthy, Juan Saucedo Figueredo, James R Goodhand, Tariq Ahmad, Nicholas A Kennedy*. IBD Service, Royal Devon and Exeter NHS Foundation Trust, Exeter, UK

\subsection{6/gutjnl-2019-BSGAbstracts.339}

Introduction Iron deficiency anaemia (IDA) commonly complicates gastrointestinal disease and impairs quality of life (QoL). Intravenous iron therapy is widely used in IDA when oral iron is poorly tolerated or ineffective, and normalisation of haemoglobin improves QoL scores. We sought to define the dosing regimens of, durability of haemoglobin responses and the prevalence of adverse events to intravenous iron (III) isomaltoside (Monofer).

Methods We undertook a service evaluation of intravenous iron use in 508 outpatients (40\% male) with gastrointestinal disease treated with 648 Monofer infusions between 2014 and 2017. Demographic, diagnosis, and treatment factors including dose of Monofer used and the number of patients treated with repeat infusions were recorded form the medical record. Anaemia was defined by WHO criteria. Iron deficiency was defined as transferrin saturation $<18 \%$ and/or ferritin $<30$ $\mu \mathrm{g} / \mathrm{L}$ (ferritin $<100 \mu \mathrm{g} / \mathrm{L}$ if CRP $>5 \mathrm{mg} / \mathrm{L}$ ). We extracted laboratory results from the electronic record for baseline, 12 weeks and 52 weeks. We sought factors associated with treatment failure, defined as ongoing anaemia at 12 weeks, using logistic regression.

Results Overall, 93\% (568/613) were anaemic at baseline with median (IQR) haemoglobin of $100 \mathrm{~g} / \mathrm{L}$ (IQR 8-12). 73\% (476/ 648) had haematinics tested and 91\% [431/476] had proven IDA. Inflamatory bowel disease was the most common indication at $30 \%(193 / 648)$ infusions. A variety of dosing regimens were used: $26 \%(167 / 648)$ received fixed dosing of $1 \mathrm{~g}, 25 \%(163 /$ 648) were dosed according to the Ganzoni formula, 14\% (91/ 648) had the dose calculated by the Ganzoni formula but limited to a single $20 \mathrm{mg} / \mathrm{kg}$ infusion, $8 \%(54 / 648)$ were dosed by the simplified dosing table and $27 \%(173 / 648)$ other dosing strategies. 74\% (479/648) infusions had follow-up haemoglobin measured 6 to 18 weeks post-infusion. The median change in haemoglobin between baseline and -8 weeks was $18 \mathrm{~g} / \mathrm{L}$ (IQR 9). 42\% (186/438) of previously anaemic patients had normalised their haemoglobin by this time. Factors associated with failure to normalise haemoglobin were male sex (odds ratio (OR) 2.9 [95\%CI 1.-.3]), age $\geq 65$ years (OR 3.4 [95\%CI 2.-.0]), higher comorbidity (OR 3.7 [95\%CI 2.-.5]) and under-dosing versus Ganzoni-calculated dose (OR 4.8 per gram underdosed [95\%CI 2.-0]). Only, 31\% (44/143) patients whose haemoglobin normalised at week 12 had recurrent anaemia at 1 year. Adverse events were rare: only one patient had a probable complement activation-related pseudoallergy that was mitigated by slowing infusions, and there were no anaphylactic reactions.

Discussion A wide-variety of dosing strategies are used in our trust. Treatment failure was associated with under-dosing , age, sex and comorbidity. Adverse events were rare. 